\title{
Remission eines komplexen periodischen katatonen Syndroms unter Elektrokonvulsionstherapie
}

\author{
Robert Queissner (D) - Walter Wurm · Christoph Ebner · Eva Reininghaus · Hans-Peter Kapfhammer
}

Eingegangen: 17. November 2017 / Angenommen: 8. September 2018 / Online publiziert: 19. September 2018

(c) Der/die Autor(en) 2018

\begin{abstract}
Zusammenfassung In diesem Fall handelt es sich um ein spontan aufgetretenes komplexes katatones Syndrom, welches sich bei einer 52-jährigen Patientin erstmals in Folge einer schweren depressiven Episode manifestierte. Es zeigte sich eine zunächst sehr ausgeprägte hypokinetisch-katatone Symptomatik, die aufgrund ihrer unterschiedlichen Qualitäten wie Gedächtnis- und Orientierungsstörungen, sowie psychotischen Symptome auch die Verdachtsdiagnose einer neurodegenerativen Erkrankung wie z.B. einer Lewy-Body Demenz oder einer Creutzfeld-Jacob Erkrankung ermöglichten. Die Patientin zeigte auf keine angewandte medikamentöse Therapie eine ausreichende Wirkung oder zeigte vor dem Erreichen einer möglichen Wirkung eine gesteigerte Empfänglichkeit für nicht tolerierbare Nebenwirkungen, die $\mathrm{zu}$ einem Absetzen des Medikaments führten. Nach erfolgter somatischer Diagnostik, die jedoch kein entsprechendes Korrelat zum Vorschein brachte, wurde eine EKT-Therapie eingeleitet, auf welche die $\mathrm{Pa}$ tientin teilremittierte und im weiteren Verlauf eine zykloide katatone Symptomatik zeigte, die stark vom Intervall zwischen den einzelnen EKT-Behandlungen abhängig war. Nach Etablierung eines ausreichenden Therapieintervalls war die Patientin vollremittiert.
\end{abstract}

Schlüsselwörter Elektrokonvulsionstherapie · Katatonie $\cdot$ Biologische Therapieverfahren $\cdot$ Neuropsychiatrie

Dr. R. Queissner $(\bowtie) \cdot$ Dr. W. Wurm · Dr. C. Ebner

Prof. Dr. E. Reininghaus · Prof. DDr. H.-P. Kapfhammer

Universitätsklinik für Psychiatrie und Psychotherapeutische

Medizin, Medizinische Universität Graz,

Auenbruggerplatz 31, 8036 Graz, Österreich

robert.queissner@medunigraz.at

\section{Remission of a complex periodic catatonic syndrome under electroconvulsive therapy}

Summary This article is reporting about a spontaneous occurred catatonic syndrome in a 52 years old female patients with no prior psychiatric illness record. The catatonia followed a severe depressive episode with psychotic symptoms. At the beginning additionally to the catatonic-symptoms severe disorientation and memory disturbances were prominent in a way it can be seen in neurodegenerative diseases like Lewy-Body-Dementia and Creutzfeldt-Jacob-Disease. The patient didn't respond on any medication or showed severe side-effects which led to discontinue the medication. After applying widespread somatic diagnostics, which has excluded a neurodegenerative disease a electroconvulsive therapy was applied. During this treatment the patient showed a recurrence of her catatonic symptoms but they remitted if there was a too long period between the convulsive treatments. After establishing a sufficient period between the convulsive treatments the symptoms remitted totally.

Keywords Electroconvulsive therapy · Catatonia · Biological treatment method $\cdot$ Neuropsychiatry

Der Begriff der Katatonie bezeichnet einen Symptomenkomplex vorwiegend psychomotorischer Symptome, welche als geschlossene konzeptuelle Entität erstmals von Karl Kahlbaum 1874 beschrieben wurde [1]. In der gegenwärtigen Literatur werden eine Vielzahl unterschiedlicher Symptome wie z. B. Ausdrucksmanierismen, motorische Spannungszustände, Befehlsautomatie, Echophänomene, Katalepsie etc. [2] zu diesem Symptomkomplex gezählt. 
Im gegenständlichen Fall handelt es sich um eine zum Zeitpunkt des Erstkontaktes 52-jährige Frau. Sie war bisher durchwegs in ihrer Heimatstadt wohnhaft, verheiratet, 1 Sohn. Sie war als Drogistin berufstätig und befand sich zuvor nie in psychiatrischer Behandlung. Ebenfalls waren keine somatischen Komorbiditäten bekannt.

Die Patientin war am 25.01.2016 das erste Mal an der Akutambulanz der Univ.-Klinik für Psychiatrie und Psychotherapeutischer Medizin vorstellig. Dort berichtete die Patientin über eine zunehmende depressive Verstimmung seit Dezember 2015. Weiters bemerkte sie bei sich ausgeprägte Konzentrationsund Gedächtnisstörungen sowie Schlafstörungen. Im psychopathologischen Befund wurden ferner überwertige bis wahnhafte Verarmungs- und Verschuldungsideen erhoben. Die beschriebenen kognitiven Defizite waren auch in der klinischen Untersuchung zu erheben. Die Patientin wirkte deutliche verlangsamt und die Konzentrationsfähigkeit war deutlich erschwert. Es wurde zunächst die Diagnose einer rezidivierenden depressiven Störung mit einer gegenwärtigen schweren depressiven Episode mit psychotischen Symptomen diagnostiziert. Aufgrund einer zum damaligen Zeitpunkt fehlenden Aufnahmekapazität wurde die Patientin zunächst an eine andere psychiatrische Abteilung im Landeskrankenhaus Süd/West Standort Süd zur dortigen stationären Aufnahme und Behandlung überstellt.

Dort zeigte sich zunächst eine Zunahme der depressiven Symptome. Die Patientin entwickelte zudem wahnhafte Verfolgungs- und Vergiftungsideen, die sie auf das Pflegepersonal als auch auf Mitpatientinnen bezog. Schlussendlich musste die Patientin gegen ihren Willen in den Unterbringungsbereich überstellt werden. Dort kam es erneut zu einer Verschlechterung des klinischen Bildes. Die Patientin zeigte eine zeitliche, örtliche wie auch situative Desorientiertheit. Sie fiel durch unorganisiertes Verhalten mit zeitweisen Fehlhandlungen auf (z.B. Blumenvase als Trinkgefäß verwenden, Fäkalienschmieren). Die antipsychotische Therapie wurde im Verlauf von Aripiprazol auf Risperidon und schließlich auf Haloperidol umgestellt. Als antidepressive Therapie wurde bei der Patientin Citalopram i.v. in zuerst niedriger Dosierung etabliert. Im weiteren Verlauf entwickelte sich bei der Patientin ein stuporöses Zustandsbild mit einer ausgeprägten Erhöhung des Muskeltonus am ganzen Körper. In der neurologischen Untersuchung zeigte sich, zusätzlich zu der beschriebenen generalisierten Tonuserhöhung, ein Zahnradphänomen betont auf die oberen Extremitäten sowie abgeschwächte Reflexe. Der restliche neurologische Status war, soweit prüfbar, unauffällig. Eine durchgeführte MRT des Schädels sowie eine Liquorpunktion blieben ergebnislos. Im EEG zeigte sich ein occcipitaler AlphaGrundrhythmus, epilepsietypische Graphoelemente kamen, soweit beurteilbar, nicht zur Darstellung. Es zeigten sich keine Hinweise auf fokale oder globale
Hirnfunktionsstörungen. Laborchemische Hinweise für ein malignes neuroleptisches Syndrom bestanden nicht. Es gab ebenfalls keine laborchemischen Entzündungszeichen oder Einschränkungen von Organfunktionen.

Die Patientin wurde am 11.03.2016 auf die Univ.Klinik für Psychiatrie und Psychotherapeutischer Medizin zwecks Durchführung einer Elektrokonvulsionstherapie rücküberstellt. Bei der Übernahme wurde aufgrund des gegenwärtigen Zustandes und der bestehenden Therapie mit $8 \mathrm{mg}$ Haloperidol der Verdacht eines Neuroleptika-induzierten Parkinsonoids gestellt. Die Therapie mit Haloperidol wurde abgesetzt und Biperiden $5 \mathrm{mg}$ i.v. eingeleitet. Die vorbestehende Therapie mit Haloperidol und Citalopram wurde abgesetzt, und es erfolgte eine Umstellung auf Olanzapin und Amitriptylin. Zusätzlich wurde Lorazepam eingeleitet, welches im Verlauf der Behandlung bis zu einer Dosierung von $10 \mathrm{mg}$ angewendet wurde. Es zeigte jedoch nur eine geringe Besserung des erhöhten Muskeltonus und blieb hinsichtlich des Psychosyndroms effektlos.

Aufgrund des bestehenden klinischen Bildes wurde differentialdiagnostisch die Möglichkeit des Bestehens einer neurodegenerativen Erkrankung in Betracht gezogen. Als mögliche Differentialdiagnosen kamen eine Autoimmunenzephalitis, eine Lewy-Body-Demenz als auch eine Creutzfeldt-JacobErkrankung in Betracht. Die Liquordiagnostik mit Bestimmung neurodegenerativer und autoimmun-inflammatorischer Marker (14-3-3 Protein, Amyloid, Anti-NMDA-Antikörper etc.) wurde wiederholt und blieb weiterhin negativ. Eine FDG-PET Untersuchung zeigte einen okzipitalen Hypometabolismus. Der Befund wurde jedoch aufgrund der eingeschränkten Compliance der Patientin (Augen schließen auf Aufforderung) als nur eingeschränkt beurteilbar angesehen. Die F-Dopa-Positronenemissionstomographie zeigte unspezifische Veränderungen in den Basalganglien, wobei ein Medikamenteneffekt nicht ausgeschlossen werden konnte (D2-Blockade).

Die Patientin zeigte in weiterer Folge eine Progredienz der klinischen Symptomatik bis hin zu einem annähernd kompletten Sistieren der Spontanbewegungen (Stupor) und dem Einstellen der Nahrungs- und Flüssigkeitsaufnahme, welche zuvor noch unter pflegerischer Hilfe möglich war.

Aufgrund der rapide zunehmenden Verschlechterung des Allgemeinzustandes wurde die Patientin auf die Intensivstation der Universitätsklinik für Neurologie überstellt. Dort entwickelte sie zusätzlich eine Pneumonie, welche antibiotisch therapiert werden musste. Die Ernährung der Patientin wurde durch Anlegen eines zentralvenösen Katheters sowie einer Magensonde gewährleistet. Nach auch dortigem fehlendem Hinweis einer akuten organischen Ursache wurde die Patientin auf die Universitätsklinik für Psychiatrie und Psychotherapeutische Medizin rücküberstellt. 
Der nach Rückübernahme vorgenommene Therapieversuch mit Maprotillin und Clozapin wurde wegen eines rasch einsetzenden Long-QT-Syndroms mit einer QTc-Zeit von bis zu $520 \mathrm{~ms}$ wieder beendet.

Da die Patientin auch weiterhin nicht zu selbstständiger Nahrungsaufnahme fähig war, musste eine PEGSonde angelegt werden. Nach diesem Eingriff zeigte die Patientin eine nicht auf äußerliche Faktoren zurückzuführende und über zwei Tage durchgehend anhaltende Schlaflosigkeit. Die Narkose für den Eingriff wurde mittels Propofol durchgeführt. Danach kam es zu einer spontanen, aber nur kurzeitigen Besserung der Symptome. Die Patienten reagierte wieder gerichtet auf Reize und äußerte wieder zusammenhängende Sätze, wenn auch mit bizarrem und nicht konklusivem Inhalt („Ich bin in der Hölle“, „Ihr seid nicht echt“). Dieser Zustand hielt für 3 Tage an. Die Patientin erlitt danach wieder einen Rückfall in den beschriebenen katatonen Zustand.

Aufgrund der erstmals beobachteten Reversibilität der Symptomatik trat der diagnostische Verdacht einer neurodegenerativen Genese des Krankheitsbildes in den Hintergrund. Bei dem insgesamt im Vergleich zum Aufnahmezeitpunkt an die Klinik wieder besseren Allgemeinzustand wurde das Einleiten einer elektrokonvulsiven Therapie (EKT) indiziert, da dies zuvor aufgrund der nicht gegebenen Narkosetauglichkeit nicht durchführbar war. Aus der bestehenden Literatur ist eine weitreichende Evidenz für die Wirksamkeit der EKT bei katatonen Zustandsbildern beschrieben [3-5]. Aufbauend darauf wird diese Form der Behandlung auch als State-of-the-Art Therapie im Konsensus-Statement der der Österreichischen Gesellschaft für für Neuropsychopharmakologie und Biologische Psychiatrie angeführt [6].

Bereits nach der ersten Anwendung zeigte sich eine deutliche, jedoch nur kurzzeitig anhaltende Besserung. Die Patientin war verbal zugänglich, sie war aber lediglich zur Person orientiert. Ca. $7 \mathrm{~h}$ nach der ersten Behandlung trat erneut ein Rückfall ein. Die Patientin verharrte wieder regungslos im Krankenbett.

Nach 8 durchgeführten Behandlungen war die Patientin durchgehend mobilisierbar, und es kam $\mathrm{zu}$ keinem erneuten Auftreten katatoner Zustände. Die Patientin fiel jedoch klinisch durch eine hyperthyme Stimmung mit zum Teil kindlich-läppischen Verhalten auf. Fremdanamnestisch konnte erhoben werden, dass dieses Verhalten nicht ihrem eigentlichen Naturell entspreche, und sie früher ein ruhiges, introvertiertes und angepasstes Temperament gezeigt habe. Zusätzlich zu den beschriebenen Wesensveränderungen imponierten bei der Patientin intermittierend desorganisierte oder inadäquate Verhaltensweisen und Handlungen. So rauchte die Patientin beständig und trotz mehrmaligen Aufforderungen, es zu unterlassen, auch im Krankenzimmer. Sie entkleidete sich mitten am Krankenhausgang. Weiters brannte sie, während sie vom Pflegepersonal dabei beobachtet wurde, mit einer Zigarette zielgerichtet den Boden an, um sie auszudämpfen. Wenn sie auf ihr Verhalten angesprochen wurde, konnte sie keinen Grund für diese Fehlhandlungen angeben. Sie zeigte in dieser Phase bei intakter Bewusstseinsklarheit eine nur eingeschränkte Orientierung sowie deutliche Auffassungs- und Merkfähigkeitsstörungen. Ferner meinte sie ihren Ehemann zwar zu sehen, aber nicht zu erkennen (Prosopagnosie). In weiterer Folge schilderte sie auch mehrfach die Auffassung, dass ihr Mann durch einen Doppelgänger ausgetauscht worden sei. Dieses Syndrom wird in der Literatur als Capgras-Syndrom bezeichnet [7]. Gegenwärtige wissenschaftliche Erkenntnisse liefern Anhalt dafür, dass die Prosopagnosie zum einen und das Capgras-Syndrom zum anderen unterschiedliche neurofunktionelle Anomalien aufweisen und somit begrifflich getrennt behandelt werden sollten [8].

Nach weiteren 10 EKT-Behandlungen im Verlauf von mehreren Wochen kam es zu einem Abklingen der ausgelenkten Affektlage. Es zeigte sich auch eine Besserung in den noopsychischen Funktionen. Die Patientin wirkte ruhig und zurückgezogen. Frau B. hatte an die letzten Monate und vor allem an die Zeit ihrer schweren Symptome keine Erinnerung.

Im anschließenden Abschnitt ihrer Erkrankung und deren Behandlung traten bei der Patientin fluktuierende Phasen von Angst und Desorientierung auf. Diese setzten schlagartig ein, wobei sich sowohl ihre Gesichtsmimik als auch der Tonfall ihrer Stimme umgehend veränderten. In diesen Situationen weinte die Patientin stark. Sie meinte nicht mehr zu existieren und hatte keine Orientierung. Ihre eigentlich sonore tiefere Stimme wechselte dabei in ein hohes, annähernd ohrendbetäubendes Kreischen, welches von ihren Angehörigen bisher nie von ihr vernommen worden wäre. Diese Phasen traten in unregelmäßigen Abständen sowohl zuhause als auch in der Klinik auf. Sie konnten auch klinisch beobachtet und dokumentiert werden. Nach dem Abklingen dieser Symptomatik hatte die Patientin keine Erinnerung mehr an diese Zeitspanne.

Zusätzlich zu der beschriebenen psychotischen Symptomatik konnten bei der Patientin reversible und von den oben beschriebenen psychomotorischen $\mathrm{Zu}$ ständen auch andere Symptome wie Hinken, Skoliose und Tortikollis beobachtet werden. Der beobachtete Verlauf erstreckt sich vom Zeitpunkt der ersten Kontaktes (01/2016) bis Oktober 2017.

Die Patientin wurde schlussendlích auf eine medikamentöse Therapie aus Venlafaxin und Quetiapin eingestellt. Die Dosierung wurde im Zuge der Behandlung mehrfach adaptiert. Sie verhielt sich bei Venlafaxin im Bereich von 150-300 mg und bei Quetiapin bei 200-500 mg in retardierter Formulierung.

Hinsichtlich des zeitlichen Verlaufs wurde jeweils eine deutliche Wiederzunahme der Symptome beobachte, je länger die letzte EKT-Behandlung zurücklag.

Zusammenfassend wurde bei der Patientin zunächst eine EKT-Serie (Behandlungen jeden 2. Tag) 
von 14 Sitzungen durchgeführt. Danach wurde auf ein Erhaltungsregime mit einem zweiwöchigen Intervall im Sinne einer ambulanten Erhaltungs-EKT umgestellt. Im weiteren Verlauf konnte das Intervall auf sechs Wochen ausgedehnt werden.

Zum Zeitpunkt Oktober 2017 ist die Patientin vollremittiert. Es traten seither kein weiterer zeitweiser Verlust der Orientierungsfähigkeit und auch keine psychotischen Symptome mehr auf. Bei kürzeren Intervallen der ambulanten EKT-Behandlungen berichtete die Patientin über gelegentliche Konzentrationsstörungen, gegenwärtig seien diese aber im Hintergrund.

Im genannten Zeitraum wurden zwei weitere MRT des Neurokraniums, sowie wie ein FDG- und ein F-Dopa-PET durchgeführt. Weiters 3 EEG-Kontrollen. Es fanden sich in keiner der Untersuchungen Änderungen zum Vorbefund.

Diagnostisch stellt der Fall ein Grenzgebiet der bestehenden Klassifikationen (DSM-V und ICD-10) dar. Grundsätzlich könnten die allgemeinen diagnostischen Kriterien einer katatonen Schizophrenie (nach ICD-10) als gegeben angesehen werden. Es bestanden anhaltende bizarre Wahngedanken sowie Wahnwahrnehmungen, katatone Symptome und Negative-Symptome wie Apathie und inadäquate Affektlage. Jedoch zeigen sowohl der klinische Gesamteindruck als auch die bis zum Beginn der Symptome blande psychiatrische Anamnese und das gegenwärtige Alter der Patientin einen für eine Schizophrenie untypischen Verlauf.

Aufgrund der unspezifischen diagnostischen Symptomkonstellation dieses Falles stellt sich die Frage, wie dieser diagnostisch und psychopathologisch eingeordnet werden sollte.

Die vermutlich ausführlichste systematische Beschreibung solcher psychopathologischer Komplexe kann den Schriften des deutschen Psychiaters Karl LEONHARD entnommen werden. In seinem psychopathologischen Konzept der endogenen Psychosen konnten sowohl die „Motilitätspsychose“ als auch die „periodische Katatonie“ als mögliche Differenzialdiagnose in Betracht gezogen werden. Während erste als Teil der zykloiden Psychosen mit einem ausgeprägten affektiven Anteil beschrieben wurde, wurde die periodische Katatonie von LEONHARD als Teil der unsystematischen Schizophrenien angesehen [9], wo jedoch die affektive Komponente als eher hintergründig angesehen wurde. Aber auch jene beschriebenen Entitäten decken den beschriebenen Fall nicht zur Gänze ab. Die Patientin wurde unsererseits diagnostisch als eine „komplexe periodische Katatonie“ abgeschlossen, da in Zusammenschau der bestehenden anamnestischen Informationen der affektive Eindruck deutlich vorrangig war und die Patientin im Vorfeld nie schizophreniforme psychotische Symptome zeigte.
Abschließend sei bei diesem Fall auf die eindrückliche körperliche Symptomatik hingewiesen, die in mehreren Stadien den Krankheitsverlauf beobachtbar bestimmte. In den Intervallen mit ausgeprägter Symptomintensität zeigte die Patientin eine multiple Unverträglichkeit auf mehrere Pharmaka sowie eine Tendenz zu gesteigerter Nebenwirkungssensitivität, vor allem hinsichtlich extrapyramidalmotorischer Symptome unter Antipsychotika sowohl der 1. als auch der 2. Generation, wie sie ansonsten bei neurodegenerativen Erkrankungen (z.B. insbesondere bei Lewy-Body-Demenz) zu beobachten sind. Eine wiederholte differentialdiagnostische Abklärung mittels spezieller Neuroimaging-Untersuchungen in diese Richtung blieb jedoch stetig ergebnislos. Auch der bisherige klinische Verlauf mit einer vollständigen Remission sprach gegen eine progrediente neurodegenerative Erkrankung.

Funding Open access funding provided by Medical University of Graz.

Interessenkonflikt R. Queissner, W. Wurm, C. Ebner, E. Reininghaus und H.-P. Kapfhammer geben an, dass kein Interessenkonflikt besteht.

Open Access Dieser Artikel wird unter der Creative Commons Namensnennung 4.0 International Lizenz (http:// creativecommons.org/licenses/by/4.0/deed.de) veröffentlicht, welche die Nutzung, Vervielfältigung, Bearbeitung, Verbreitung und Wiedergabe in jeglichem Medium und Format erlaubt, sofern Sie den/die ursprünglichen Autor(en) und die Quelle ordnungsgemäß nennen, einen Link zur Creative Commons Lizenz beifügen und angeben, ob Änderungen vorgenommen wurden.

\section{Literatur}

1. Kahlbaum K. Die Katatonie oder das Spannungsirresein. 1.Aufl. Berlin:August Hirschwald; 1874.

2. Hoff P. Der katatone Symptomenkomplex in der Systematik Kraepelins. In: Bräunig P, Hrsg. Differenzierung katatoner und neuroleptika-induzierter Bewegungsstörungen. 1.Aufl. Stuttgart: Thieme; 1995.

3. Unal A, Altindag A, Demir B, Aksoy I. The use of lorazepam and electroconvulsive therapyin the treatment of catatonia: treatment characteristics and outcomes in 60 patients. JECT. 2017;33(4):290-3.

4. Weiner R, Reti I. Key updates in the clinical application of electroconvulsive therapy. Int Rev Psychiatry. 2017;54(2):54-62.

5. Fink M, Kellner C, McCall W. Optimizing ECT technique in treating catatonia. JECT. 2016;32(3):149-50.

6. Kasper S, Sachs G, Bach M, Erfurth A, Frey R, Fruhwürth G, et al. Schizophrenie Medikamentöse Therapie. KonsensusStatement - State of the art 2016. CliniCum Neuropsy. 2016;1:12-20.

7. ForstlH,AlmeidaO, OwenA, BurnsA,HowardR.Psychiatric, neurological and medical aspects of misidentification syndromes: a review of 260 cases. Psychol Med. 1991;21:905-10.

8. Ellis H, Lewis M. Capgras delusion: a window on face recognition. Trends Cogn Sci. 2001;5:149-56.

9. Leonhard K. Aufteilung der endogenen Psychosen und ihre differenzierte Ätiologie. 7. Aufl. Stuttgart:Thieme; 1995. 